\title{
Experimental Evaluation of Odor Treatment for Discharge Plasma by Using Ceramic Tube
}

\author{
Hee Kyu Lee, Dorjsuren Baatarkhuu, Ulziikhutag Ganbat \\ School of Engineering, Mongolian University of Life Sciences(MULS), Ulaanbaatar, Mongolia
}

Email address:

heek1@hanmail.net (H. K. Lee), baatar_khuu2000@yahoo.com (D. Baatarkhuu), ulganbat67@yahoo.com (U. Ganbat)

To cite this article:

Hee Kyu Lee, Dorjsuren Baatarkhuu, Ulziikhutag Ganbat. Experimental Evaluation of Odor Treatment for Discharge Plasma by Using Ceramic Tube. International Journal of Energy and Power Engineering. Vol. 4, No. 5, 2015, pp. 322-326. doi: 10.11648/j.ijepe.20150405.23

\begin{abstract}
The odor removal technology in this research is the new technology which emits the discharge plasma in the area of $\mathrm{AC}$, and through the research discharge plasma-ozone-absorption filter is accomplished as process system. In order for this, we combine the advantages of chemical technology, a discharge plasma technology, and ozone absorption filter technology together and emit the plasma on the odor materials that is collected in first step. Through this process, we are able to see that hydrogen sulfide, ammonia, mercaptan, trimethylamine and volatile organic compounds, bacteria, etc. which are major cause of various ill odor are removed, and through passing the plasma generator which makes free radicals that is ten times more powerful than ozone sterilizer, the rest of the odor substances is removed by redox reactions. In the third step, the rest of zone is absorbed and emitted into the air by using the absorption filter. The rate of efficiency as the result of this research is $99 \%$, using $5 \mathrm{~m}^{3} / \mathrm{min}$ ceramic tube and applying $15 \mathrm{~m} / \mathrm{sec}$ wind and $360 \mathrm{~mm} /$ Aq pressure.
\end{abstract}

Keywords: Discharge Plasma, Odor Treatment, Ceramic Tube, Ozone Control, Bacteria, Volatile Organic Compounds, Air Pollution

\section{Introduction}

Plasma relation techniques are come, being applied from the environmental contamination control technical background including air pollution, ozone control etc. technique and they do not divide not to be, alias does and as Plasma comes to soak, is mixed. So there is a possibility of seeing like this name frequently from general family electrical appliance etc. But Plasma relation techniques the application field develops and environmental industry is developmental at class speed. The plasma the element inflicts the electric charge of high voltage and the high frequency in the ion and the electron moves and actively, makes and the element the active radical where the reaction is easy the ion chemically creates from and to oxidize the offensive odor substance. Therefore ozone seizure and different point ozone seizure the ozone which is an oxidizer which is powerful occurs and with the offensive odor substance the other side which gets up a reaction, plasma control offensive odor substance oneself oxidizing with radical reaction, the reaction velocity which sees happens quickly. But the problem which important the research description below for the efficiency of plasma occurrence system on a large scale is in the process of advancing and is efficiency of the plasma discharge tube, in order to increase important efficiency of the discharge tube is appearing with the variable which shape etc. of size and the electrode of quality of material and the discharge tube of form and the discharge tube of impressed voltage is.

\section{Odor Removal Procedure}

Generally, the offensive odor treatment by the offensive odor treatment and optical catalyst etc. is used as the method used as the deodorizing device or the removing apparatus to the deodorization method by the adsorption method, ozonization process, photo catalytic oxidation, scrubber washing method, the biological filter, and the plasma generation. Particularly, in the bad smell by the plasma generation apparatus or the deodorizing device, the plasma generation apparatus is obligatorily used. The bad smell using the plasma discharge till the recent and method of removing the contaminated gas including the VOCs (the Volatile Organic Compounds: volatile organic compound) etc had been being developed as various types. The plasma method developed in the initial is the contaminant of the gaseous using the plasma of the pulse corona form may be referred to the 
mode which is the mode which removes and it puts the inert gas while creating plasma using the high voltage of $10 \mathrm{kV}$ to $25 \mathrm{kV}$ in the electrode of the flat board form and pin and safely maintains the plasma state. But the form of plasma the plasma method of this kind of the form was irregularly molded and the odor removal efficiency fell down. The limit was in the management cost and processing capacity and it was unable to reach to the commercialization. As the mode in which and the plasma deodorization equipment of the other form removes the contaminant using the plasma of the dielectric barrier discharge form, this apparatus puts the dielectric barrier in the between electrode of the flat board form and the even plasma at the desired high voltage of $7 \mathrm{kV}$ to $15 \mathrm{kV}$ is formed and it reacts to the bad smell component. But the efficiency this apparatus drops on the pressure drop and electrode to the impurity deposition including carbon etc. There is a problem that it makes the efficiency of the above-described conventional method high. The dwelling time of the contaminated gas is prolonged or the electric discharge power has to authorize the high voltage of $7 \mathrm{kV}$ to $15 \mathrm{kV}$.

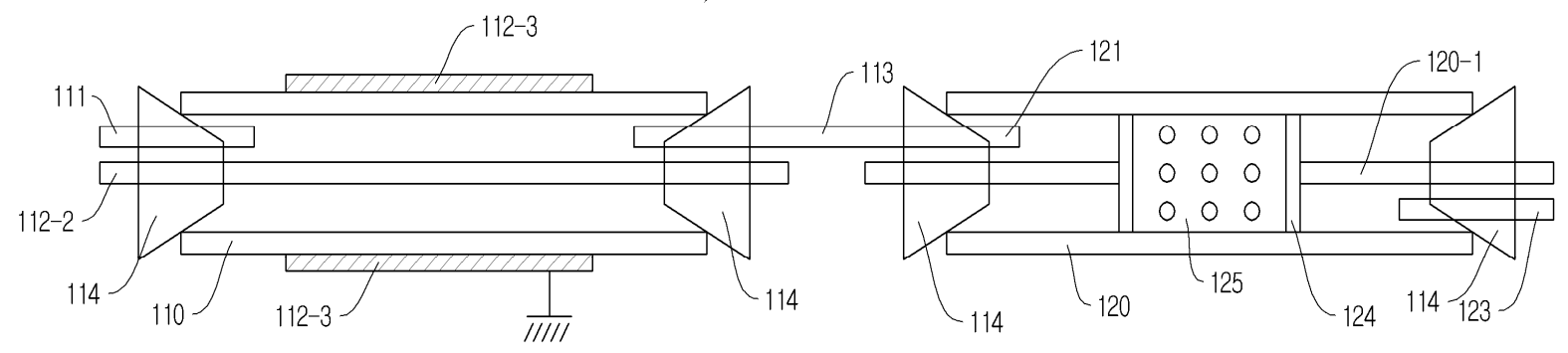

Fig. 1. Schematic diagram of odor removing apparatus.

In Fig. 1, the gas containing the plasma generation part (110) is the contaminated fume from the outside, for example, acetaldehyde comprises the first outlet for gas (113) discharging gas in which the plasma generating unit (112), which incinerates the bad smell component included in the gas flowed in through the first gas inlet (111) and removed and bad smell component are removed with plasma generated by the first gas inlet (111), flowed in and high voltage.

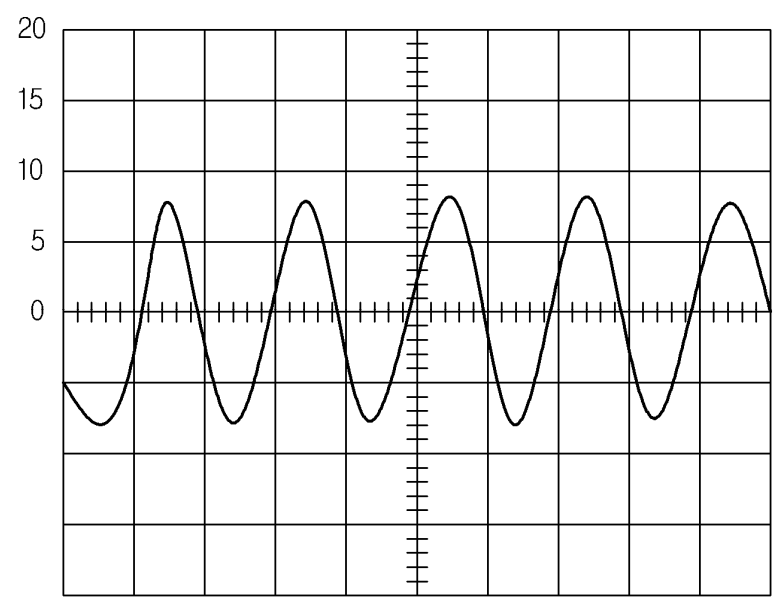

Time(25 $\mu \mathrm{s} /$ div $)$, voltage( $5 \mathrm{kV} / \mathrm{div}), 25 \mathrm{kHz}$

Fig. 2. Waveform of applied voltage Discharged power $(W)$.

Specifically, the plasma generating unit (112) includes the stand wire (112-2) which passes through the inner portion of the cylindrical plasma pipe (112-1), consisting of the acryl and plasma pipe (112-1) and in which the high voltage is applied, and the aluminum outer cover (112-3) covering the outer periphery of the plasma pipe (112-1). Here, the aluminum outer cover (112-3) serves of the ground. In the meantime, the voltage of 10 to $20 \mathrm{kV}$ can be applied at the stand wire (112-2) of the plasma generating unit. Here, the Exterior diameter of the plasma generation part materially formed with the acryl is $26 \mathrm{~mm} \varphi$, and the inner diameter is $24 \mathrm{~mm} \varphi$, and length is $250 \mathrm{~mm}$. The length of the aluminum outer cover may be $150 \mathrm{~mm}$.

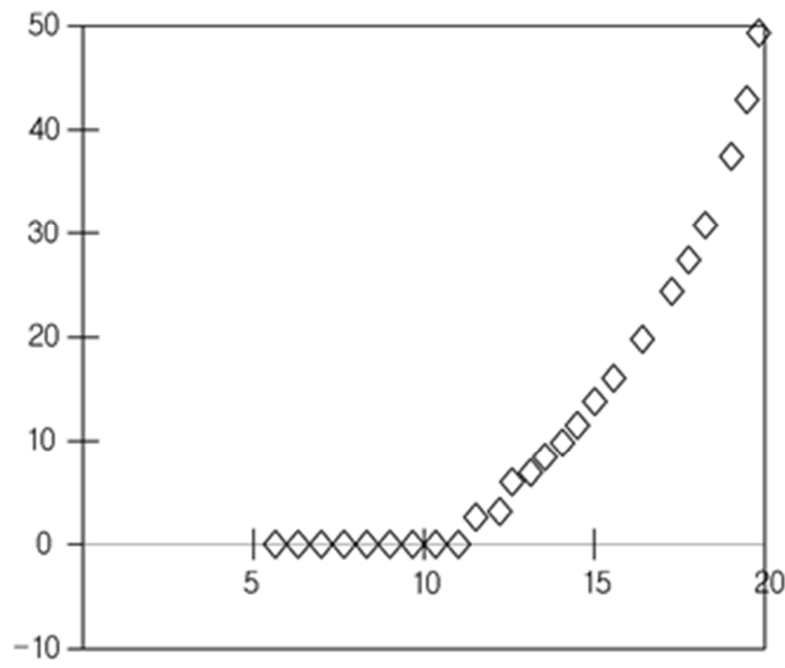

Applied voltage $(\mathrm{kV})$

Fig. 3. Plasma discharged power following applied voltage.

In Fig.2, the high voltage applied in the plasma generation part (110) may be the alternating current high voltage of $25 \mathrm{kHz}$ connecting to the power output of the stabilized DC power supply and is generated to the primary voltage of the neon inverter transformer.

it was measured through the wattmeter installed at the input voltage is the primary side of the transformer the applied 
voltage measured through the high voltage probe in the digital scope. At this time, the moisture-free gas measured while spilling the moisture-free gas of 52 / min within the bad smell component removing apparatus using the plasma discharge. The DC drive of the neon inverter transformer started the discharge in $12 \mathrm{kV}$.

Removing ratio(\%)

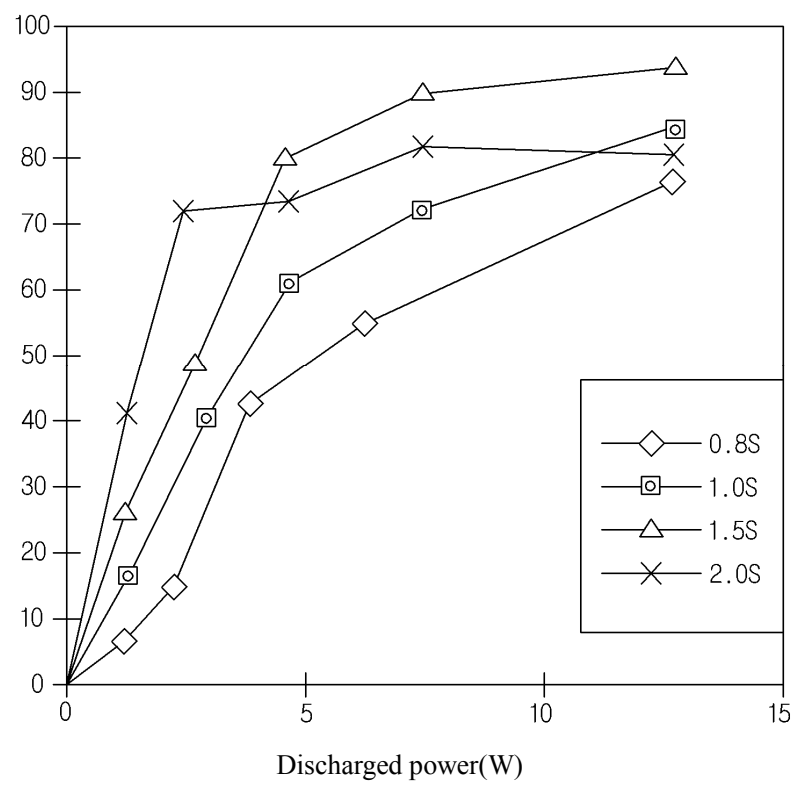

Fig. 4. Acetaldehyde removal ratio following discharged plasma power.

In Fig. 4, the removal ratio of the acetaldehyde about the plasma discharge electricity according to the dwelling time of the bad smell component within the plasma generation part could know that the degradability or the removal ratio of acetaldehyde increased as the dwelling time was long. At this time, in the dwell time is 1.5 second, it obtained with the removal ratio of the maximum $90 \%$ of acetaldehyde. It can confirm that the removal ratio makes saturation state in $6 \mathrm{w}$.

\section{Experimental Apparatus}

The experimental set up used in this study is shown in Fig.5 and Fig.6. The plasma reactor used in this study consists of ceramic tube as dielectric tube. Here appears the odor processing unit including the ground electrode in which part is wound in the exterior of the exposed implanter pole: dielectric tube from the ceramic tube in the $\mathrm{Al}$ mesh the voltage is applied it is positioned in the center of inside of the porous ceramic tube: ceramic tube in which the ozone decomposition catalyst is dipped it includes the respective dielectric tube included in both ends the gas inlet and gas outlet, and the multiple holes and the power supply unit for applying the high voltage in the implanter pole. The multiple holes is inserted inside the dielectric tube and is formed so that the odor gas pass in the inlet port the outlet port. if the high voltage is applied at the implanter pole, plasma is generated in the micro pore and the multiple holes existing in the ceramic tube.

The discharge tube used 6 ceramic tubes, the diameter of the ceramic tube was $6 \varphi$, to each ceramic tube used stainless steel of $3 \varphi$ with the electrode. The outside of the ceramic tube was covered with the aluminum mesh and covered again the whole ceramic tube with the aluminum mesh. Finally, this put in the acryl tube and fixed. The voltage adjusted $10 \mathrm{kV} 60 \mathrm{~W}$. To the discharge tube $5 \mathrm{~m}^{3} / \mathrm{min} 15 \mathrm{~m} / \mathrm{sec}$ wind velocities were authorized at control dosage of time.

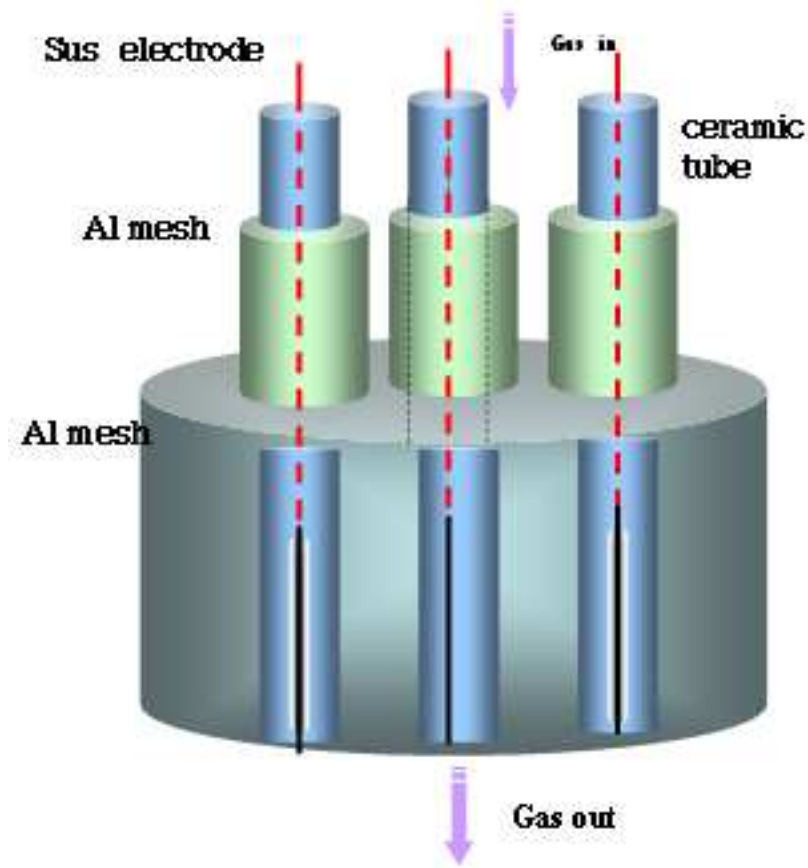

Fig. 5. Schematic diagram of discharge tube.

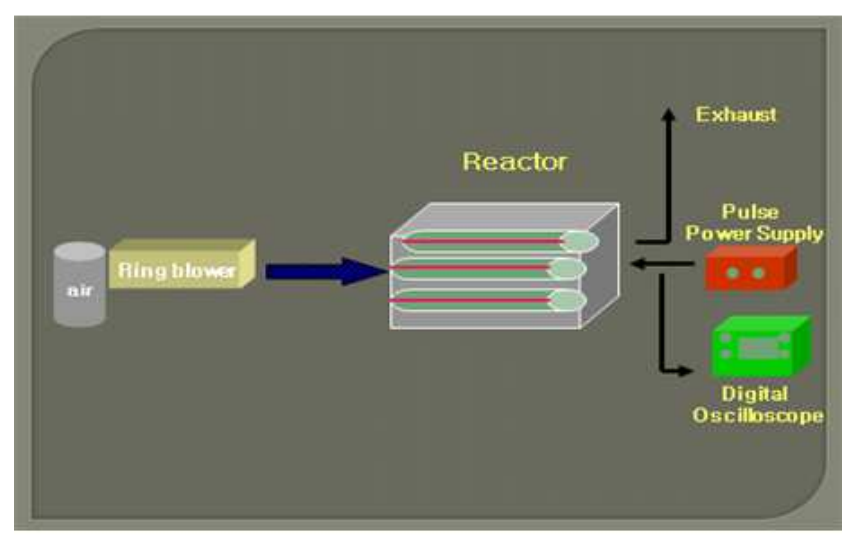

Fig. 6. Schematic diagram of experimental apparatus.

The air proximity establishes the injection nozzle at rapid pace will can jet on discharge tube entrance side of the plasma reaction apparatus inside with, from the minute description injection nozzle internal furnace discharge tube to jet the jet air which is jetted, will expand the plasma reaction territory where the contaminated gas reacts and will be able to control a reaction time enough long and there is an effect the offensive odor removal efficiency of the contaminated gas on a large scale will be able to improve. The impurity which together, contains within the plasma discharge at the time of contaminated gas (the dust, adhesiveness material etc.) 
there is a possibility also the effect will be able to minimize the arc occurrence department contamination in compliance with getting. The nozzle hole in order for at $1 \mathrm{~mm}$ to $2 \mathrm{~mm}$ degree to form with the instantaneous velocity of air to move with $10 \mathrm{~m} / \mathrm{sec}$ to $30 \mathrm{~m} / \mathrm{sec}$ very small quickly from plasma occurrence department of the discharge tube, is desirable. Like this air (the jet air) is quick according to flowing, the plasma which creates from plasma occurrence department moves with a top and expands a plasma reaction territory about the contaminated gas.

\section{Experimental Results}

From the injection nozzle the jet air which is supplied with the oxygen and the moisture in air and leads from experiment rig and reacts if the equipment which ozonator etc. for an ozone commitment is unnecessary the oxidation radical which is an oxide which is powerful (promotes $\mathrm{OH}$ radical) creations with, like existing and does not use, has the strong point.

$5 \mathrm{~m}^{3} / \mathrm{min}$ contaminated gases are controlled from this experiment, the creation of the contaminated gas which is controlled as the same numbers as Table 1 is.

In the meantime, conventionally, the electricity of being supplied since most of methods processing the odor using the high voltage use for the removal of odor like only the oxidation active species is unable to be efficiently used. On the other hand, here relates to the plasma-generating at the micro pore of the ceramic tube carrying the high specific surface area. And the catalyst surface is directly exposed to plasma and the catalyst is activated and odor is more removed. The ceramic tube catalytic reactor is semi-permanently usable. And the pressure loss is not nearly generated to the pellet filling type catalytic reactor which is generally used and the electricity of being consumed in the transfer of the gas is saved.

According to increase the supplied voltage the voltage starts from the center and the plasma generation develops into the radial direction. And on the whole, plasma is uniformly generated if the specified voltage is exceeded. The production of plasma has close related with the average magnetic field intensity. The average magnetic field intensity is increased according to the increase of the voltage since as to the reactor, the electrode spacing is fixed. And the production of plasma is developed into the radial direction.

Table 1. The creation of the contamination gas.

\begin{tabular}{lllll}
\hline element & $\mathbf{N H}_{3}$ & $\mathbf{H}_{\mathbf{2}} \mathbf{S}$ & Trimethylamine & TVOC \\
\hline Concentration $(\mathrm{ppm})$ & 100 & 100 & 100 & 100 \\
\hline
\end{tabular}

From here, TVOC was consisted of Toluene and Aldehyde etc.

In Fig.7, When plasma occurrence the voltage occurs normally from above of $5 \mathrm{kv}$ and the voltage exceeds $8 \mathrm{kV}$, NOx occurrences quantity increase and, this comes to seem in compliance with the destruction of the nitrogen in the air which is caused by with energy oversupply with the fact that occurs.

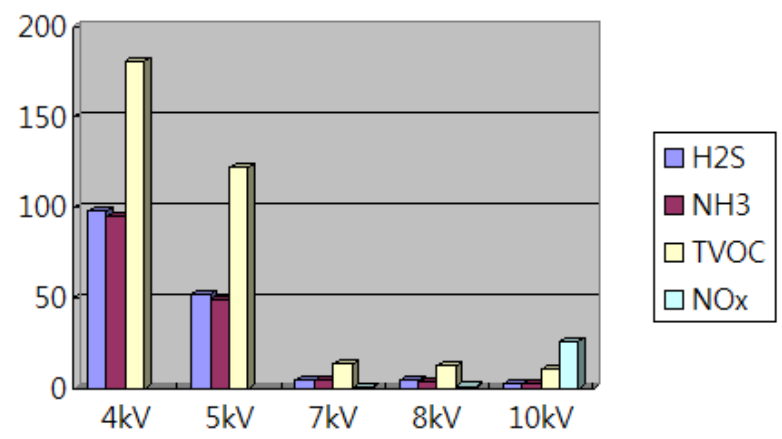

Fig. 7. Treatment result for voltage effects.

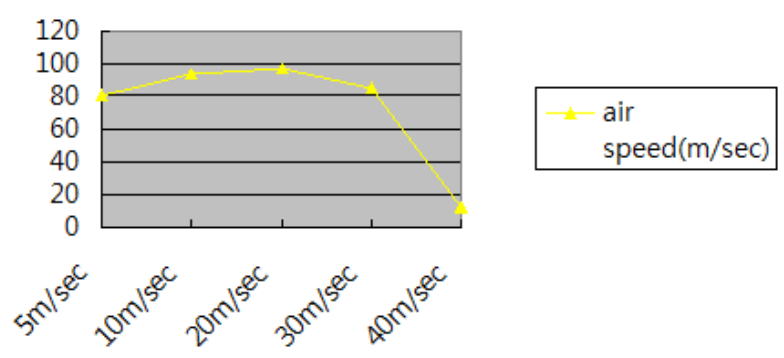

Fig. 8. Treatment ratio for applying air speed.

Fig. 8 shows the experimental result of odor treatment ratio as a function of the applied air $\operatorname{speed}(\mathrm{m} / \mathrm{sec})$. The injection nozzle expands a plasma occurrence territory and supplies helps gets up the plasma reaction which is stable influences the oxygen and the moisture and to oxidation reaction. Experimental result and the injection nozzle leads and the air which flows (the jet air) the territory of the recording plasma where the flux will be quick increases and the contaminant removal efficiency improves, to exceed $20 \mathrm{~m} / \mathrm{sec}$ to $30 \mathrm{~m} / \mathrm{sec}$ flux, the efficiency falling, starts. The efficiency suddenly falls with $30 \mathrm{~m} / \mathrm{sec}$, after that, the plasma where the flux creates too quickly spreads and does not expand a plasma territory not to be able, is thought the because being terminated early.

\section{Conclusion}

1. The ambient pressure and the characteristic in which the operation is possible and which does based on the alternating current and reducing the operate electric power have as to the removing apparatus.

2. There can be the advantage that the catalyst of the high specific surface area is directly exposed to plasma and the catalyst activation for the reducing odor and sterilization easily occurs as the plasma-generating at the micro pore of the ceramic

3. The dielectric tube as to a preferred embodiment may be formed of preferably Quartz tube, the ceramic tube etc. Pipe comprised of the dielectric meaning the comprised discharge tube and is known in the relevant industry in the ozone generating apparatus, the plasma-generating apparatus etc are selected as the material with high heat 
distortion temperature the dielectric constant is high. The ceramic tube is selected as the dielectric with high mechanical strength the Quartz tube is appropriate and it can use as the dielectric with high heat distortion temperature

\section{References}

[1] Water Environment Federation(1996). Operation of municipal waste water treatment plants.

[2] U.S. EPA(1987). Design Manual Phosphorous Removal.

[3] Metcalf \& Eddy Inc,(2003). Waste water Engineering.

[4] H.K.LEE et al., Device for removing odorous gas using plasma discharge, patent for KIPO, 10-2009-0133610, 2009.

[5] G.Dinelii, M. Rea, Pulse power electrostatic technologies for the control of flue gas emissions, J. Electrostatics 25 (1990) 23-40.

[6] C.M. nunez, G.H. ramsey, W.H. Ponder, J.H. Abbott, L.E. Hamel, P.H. kariher, Corona destruction an innovative control technology for VOCs and air toxics, Air Waste 43 (1993) 242-247.
[7] J.S.. chang, P.A. Lawless, T. Yamamoto, Corona discharge processes, IEEE Trans. Plasma Sci. 19(6) (1991) 1152-1166.

[8] M.G. Nickelsen, W.J. Cooper, C. Kurucz, T. waite, Removal of benzene and selected alkylsubstituted benzene from aqueous solution utilizing continuous electron radiation, Environ. Sci. technol. 26(1992) 144-152.

[9] S. Hosokawa, Application of Non-thermal plasma for odor control, Proceedings of the IEJ-ESA Joint Symposium on Electrostatics, 1996, pp. 41-50.

[10] Jing Li, Wanming Sun, bpashaie, S.K. Dhali, Streamer discharge simulation in flue gas, IEEE trans. Plasma Sci. 23(4) (1995) 672-678.

[11] H.H. Kim, K. tsunoda, K. Shimizu, S. tanaka, A. Mizuno, T. Yamamoto, Experimental approach to enhance efficiency of non-thermal plasma in flue gas cleaning, J. Adv, Oxid. Technol. 4(3) (1999) 347-351.

[12] K. Kinoshita, Y. Fujiyama, H.H. Kim, S. Katsura, A. Mizuno, Control of tobacco smoke and odors using discharge plasma reactor, J. Electrostatics 42 (1997) 83-91. 